LAWRENCE LIVERMORE NATIONAL LABORATORY

\title{
Performance of Rank-2 Fortran 90 \\ Pointer Arrays vs. Allocatable Arrays
}

E. Zywicz

October 11, 2005

An informal report for communication of compiler performance issues with Livermore Computing vendors. 
UCRL-TR-216197

This document was prepared as an account of work sponsored by an agency of the United States Government. Neither the United States Government nor the University of California nor any of their employees, makes any warranty, express or implied, or assumes any legal liability or responsibility for the accuracy, completeness, or usefulness of any information, apparatus, product, or process disclosed, or represents that its use would not infringe privately owned rights. Reference herein to any specific commercial product, process, or service by trade name, trademark, manufacturer, or otherwise, does not necessarily constitute or imply its endorsement, recommendation, or favoring by the United States Government or the University of California. The views and opinions of authors expressed herein do not necessarily state or reflect those of the United States Government or the University of California, and shall not be used for advertising or product endorsement purposes.

This work was performed under the auspices of the U.S. Department of Energy by University of California, Lawrence Livermore National Laboratory under Contract W7405-Eng-48. 


\section{Performance of Rank-2 Fortran 90 Pointer Arrays vs. Allocatable Arrays}

\section{Edward Zywicz}

October 11, 2005

Methods Development Group

Lawrence Livermore National Laboratory

PO Box 808, L-125

Livermore, CA 94551 
UCRL-TR-216197

\section{Introduction}

The computational performance of two-dimensional Fortran 90 arrays defined with the pointer attribute were compared to identically sized arrays defined with the allocatable attribute. The goal of this work was to quantify the computational cost of using each array type within a high-performance finite element setting.

\section{Test Program}

A test program was developed that mirrors how the main 2-D arrays are employed within the explicit finite element code DYNA3D. The test code first allocates the array (3 by $1,000,000)$ and then calls a subroutine 1000 times with the array and the array size as its calling arguments. The CPU time necessary to complete all 1000 calls was measured. The Fortran coding for each variant tested was simply:

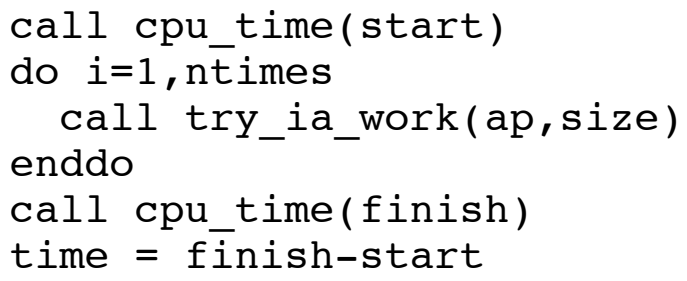

Two types of subroutines were tested. The "working" subroutine type performs operations on the array and explores both the cost of passing the array to the subroutine as well as the cost to operate on it. The main sections of these subroutines contain the coding:

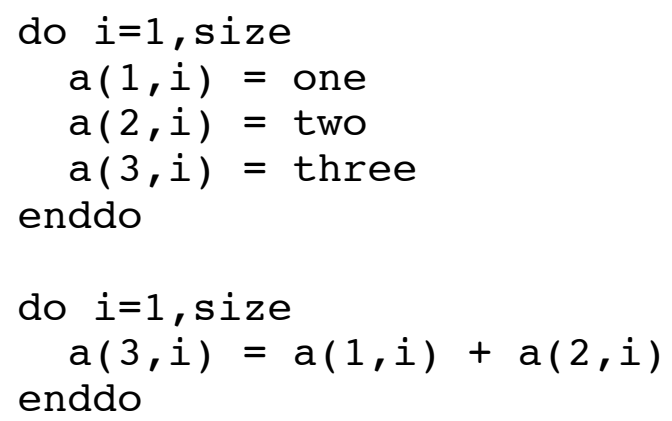

The "passing" type of subroutines merely adds the first two numbers of the array together and returns. It is intended to explore the cost of passing the array to the subroutine, and their main sections look like:

$$
\begin{aligned}
& a(1,1)=\text { one } \\
& a(2,1)=a(1,1)+\text { one }
\end{aligned}
$$


Various combinations of array types, subroutine types, subroutine interfaces, and calling statements were explored. In the main calling routine, the main array had one of two attributes - allocatable or pointer. In the subroutine, the main array had a declared size and, in some cases, was assigned the pointer attribute. In general, the array was passed with an implicit range (call try (a, nsize)); however, in some cases, the array range was explicitly specified (call $\operatorname{try}(\operatorname{a}(1: 3,1:$ Nsize) )). When admissible, both explicit and implicit interfaces were tested; when the array in the subroutine was assigned the pointer attribute, only explicit interfaces are permitted. Both working and passing types of subroutines were tested for all permutations. Table 1 summarizes the 14 different combinations considered.

Table 1: Permutations Examined

\begin{tabular}{|l|l|l|l|l|l|}
\hline Case \# & $\begin{array}{l}\text { Main Routine - } \\
\text { Array Attribute }\end{array}$ & $\begin{array}{l}\text { Subroutine - } \\
\text { Array Attribute }\end{array}$ & $\begin{array}{l}\text { Subroutine } \\
\text { Type }\end{array}$ & Interface & $\begin{array}{l}\text { Range } \\
\text { Specified }\end{array}$ \\
\hline 1 & Allocatable & None & Working & Implicit & No \\
\hline 2 & Allocatable & None & Passing & Implicit & No \\
\hline 3 & Allocatable & None & Working & Explicit & No \\
\hline 4 & Allocatable & None & Passing & Explicit & No \\
\hline 5 & Pointer & None & Working & Implicit & No \\
\hline 6 & Pointer & None & Passing & Implicit & No \\
\hline 7 & Pointer & None & Working & Explicit & No \\
\hline 8 & Pointer & None & Passing & Explicit & No \\
\hline 9 & Pointer & None & Working & Implicit & Yes \\
\hline 10 & Pointer & None & Passing & Implicit & Yes \\
\hline 11 & Pointer & None & Working & Explicit & Yes \\
\hline 12 & Pointer & None & Passing & Explicit & Yes \\
\hline 13 & Pointer & Pointer & Working & Explicit & No \\
\hline 14 & Pointer & Pointer & Passing & Explicit & No \\
\hline
\end{tabular}

Contained in the Appendix is the full source code listing for the test program.

\section{Results}

The program was compiled and run on four different platforms using their default Fortran compiler. The four platforms tested were:

1) Krakov (SGI R14,000) using f90 (version 7.4.2m) with -O3 -mips4 -64,

2) ILX (Intel Xeon) using ifort (version 8.0) with -O3,

3) GPS (Compaq Tru64) using f90 with -fast,

4) UP (IBM Power5) using xlf90 with -O4.

Table 2 contains the average CPU times from three runs on each platform.

Table 2: CPU times (seconds - average of 3 runs) 


\begin{tabular}{|c|c|c|c|c|}
\hline Case \# & SGI - Krakov & Intel Xeon - ILX & Compaq - GPS & IBM Power5 - uP \\
\hline 1 & 40.5 & 25.3 & 18.9 & 1.14 \\
\hline 2 & $1.21 \mathrm{e}-4$ & 0 & 0 & 0 \\
\hline 3 & 40.6 & 26.4 & 19.0 & 1.16 \\
\hline 4 & $1.24 \mathrm{e}-4$ & 0 & 0 & 0 \\
\hline 5 & 40.5 & 26.0 & 18.9 & 1.16 \\
\hline 6 & $1.31 \mathrm{e}-4$ & 0 & 0 & 0 \\
\hline 7 & 40.5 & 27.34 & 18.9 & 1.16 \\
\hline 8 & $1.57 \mathrm{e}-4$ & 0 & 0 & 0 \\
\hline 9 & 168 & 25.6 & 18.9 & 5.88 \\
\hline 10 & 129 & 0 & 0 & 4.77 \\
\hline 11 & 169 & 25.4 & 18.9 & 5.85 \\
\hline 12 & 129 & 0 & 0 & 4.79 \\
\hline 13 & 36.2 & 24.8 & 25.8 & 3.53 \\
\hline 14 & $1.32 \mathrm{e}-4$ & 0 & 0 & 0 \\
\hline
\end{tabular}

\section{Analysis of Results}

\section{SGI platform:}

The results for the SGI suggest that as long as you do not explicitly declare the array range on the calling line (cases 9-12), the performance is not significantly impacted by what attributes an array has or how it is passed to a subroutine (cases 1-8; 13-14). However, arrays declared with the pointer attribute and passed with an explicit interface (cases 13-14) perform slightly better, $10 \%$ faster. Given the drastic increase in CPU time when the array ranges are explicitly specified on the calling line (cases 9-12), one might assume that a "copy-in/copy-out" operation is being performed. Clearly, specifying the array ranges should be avoided when possible.

\section{Intel Xeon platform:}

The performance on the Intel platform is completely independent of the coding style or attributes used. Unfortunately, the averaged results summarized in Table 2 are somewhat misleading due to machine utilization. Often, one of the three values averaged was $15 \%$ to $30 \%$ larger than the other two, and this variation causes the averaged values to vary a bit. However, based upon the minimum time for each case, no appreciable difference exists between the cases examined.

\section{Compaq Tru64 platform:}

There is no difference in performance with coding style or attributes used, except when the array has the pointer attribute in both the main routine and the subroutine (cases 13$14)$. In the later case there is a marked degradation in performance $(\sim 37 \%)$. 


\section{IBM Power5 platform:}

On the IBM, as long as you do not explicitly declare the array range on the calling line (cases 9-12) or assign the pointer attribute to the array in the subroutine (cases 13-14), the performance is independent of the coding style and array attributes used in the main routine (cases 1-8). Like the SGI, arrays passed with their ranges explicitly declared are appreciably slower, and this suggests that a "copy-in/copy-out" operation is occurring. Furthermore, like the Compaq, a substantial slow down (3 times) occurs when the array in the subroutine is assigned the pointer attribute (cases 13-14).

\section{Conclusions}

In general, explicitly declaring the array ranges in a call statement should be avoided since it can cause a substantial degradation in performance on some platforms (e.g., SGI and IBM Power5) and offers no performance benefits. Unfortunately, based upon this work, no conclusions can be drawn about which array attributes are best to use within a finite element framework due to vendor variations in the Fortran compilers. The consistent use of arrays with the pointer attribute (i.e., in the main and all subsequent subroutines) show marked degradation on select platforms. For example, on the IBM they are 3 times slower other attribute combinations. This is a shame since the consistent utilization of pointer arrays is highly desirable within a finite element code, i.e., they do not have the same language limitations imposed on them as allocatable arrays. 
UCRL-TR-216197

\section{Appendix}

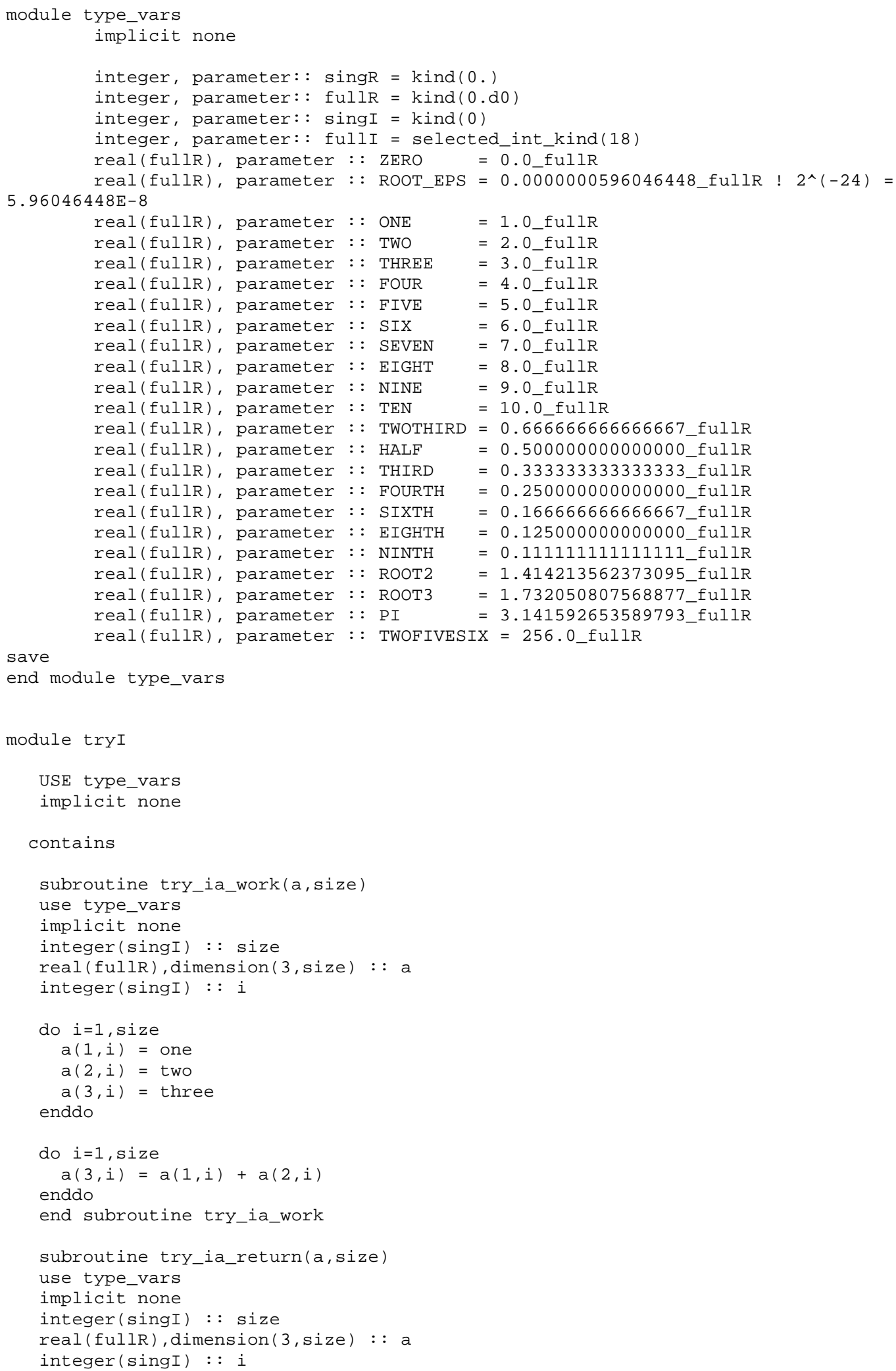




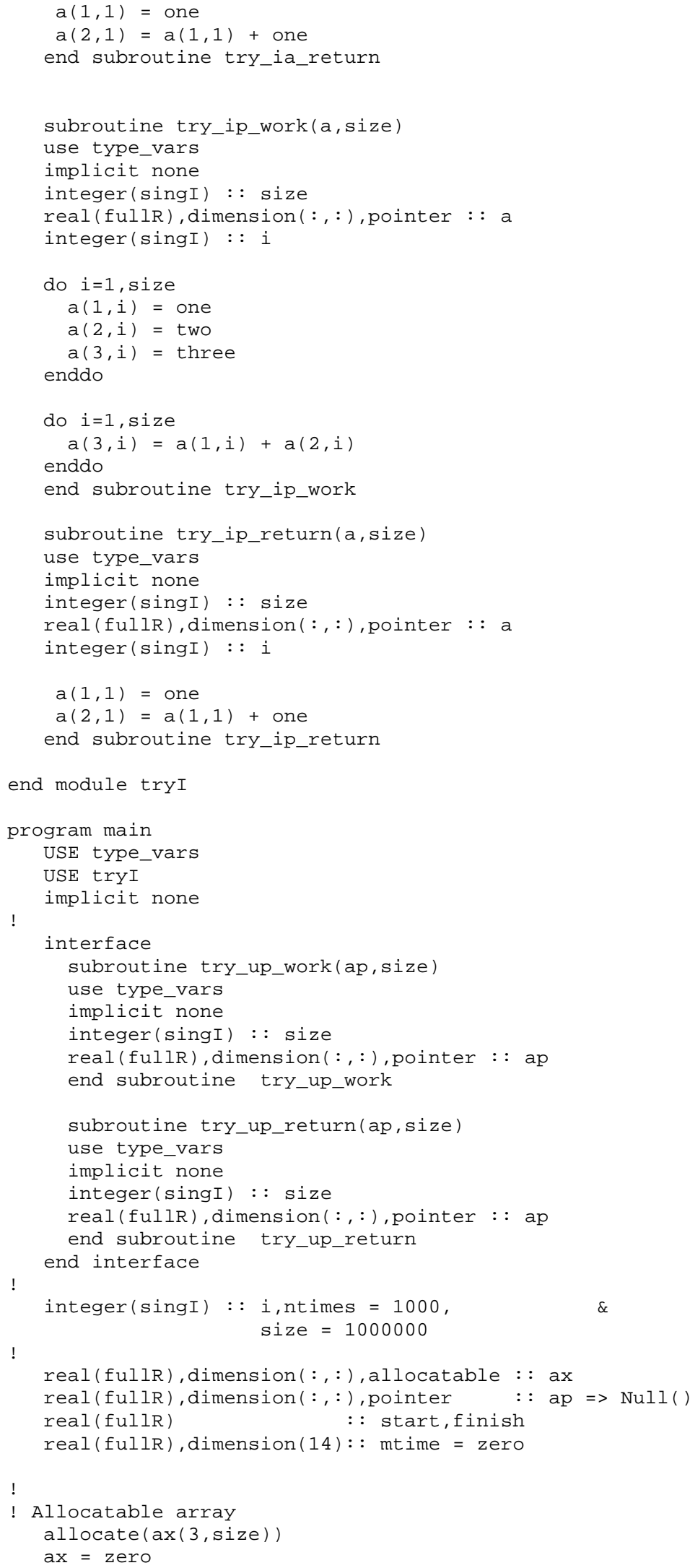




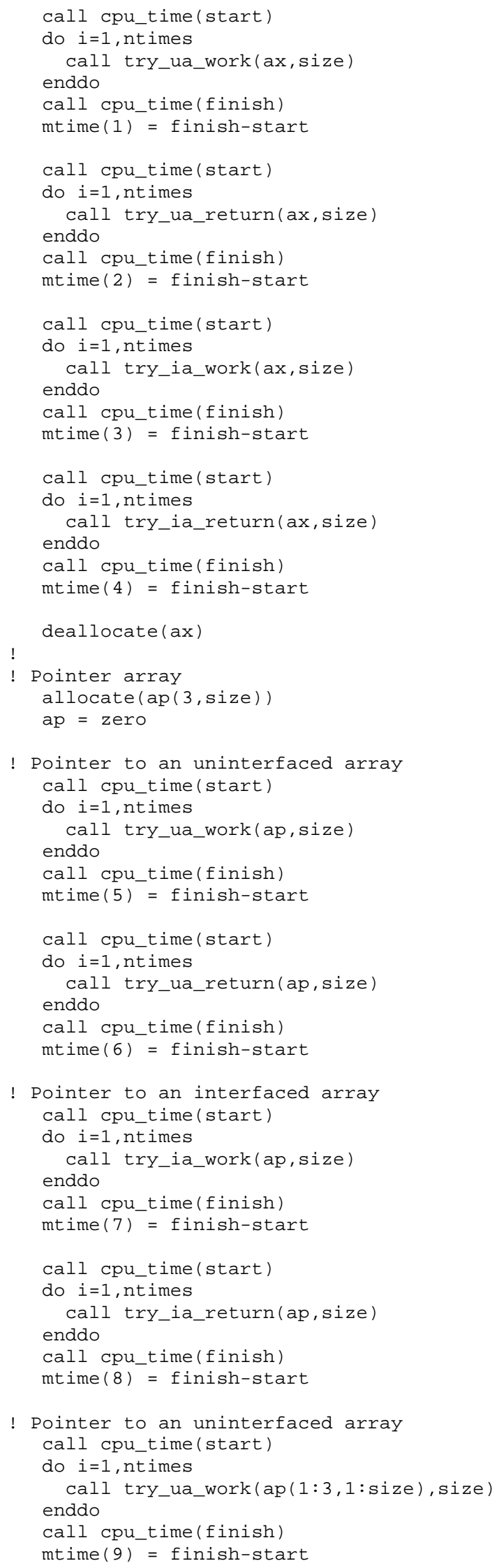


UCRL-TR-216197

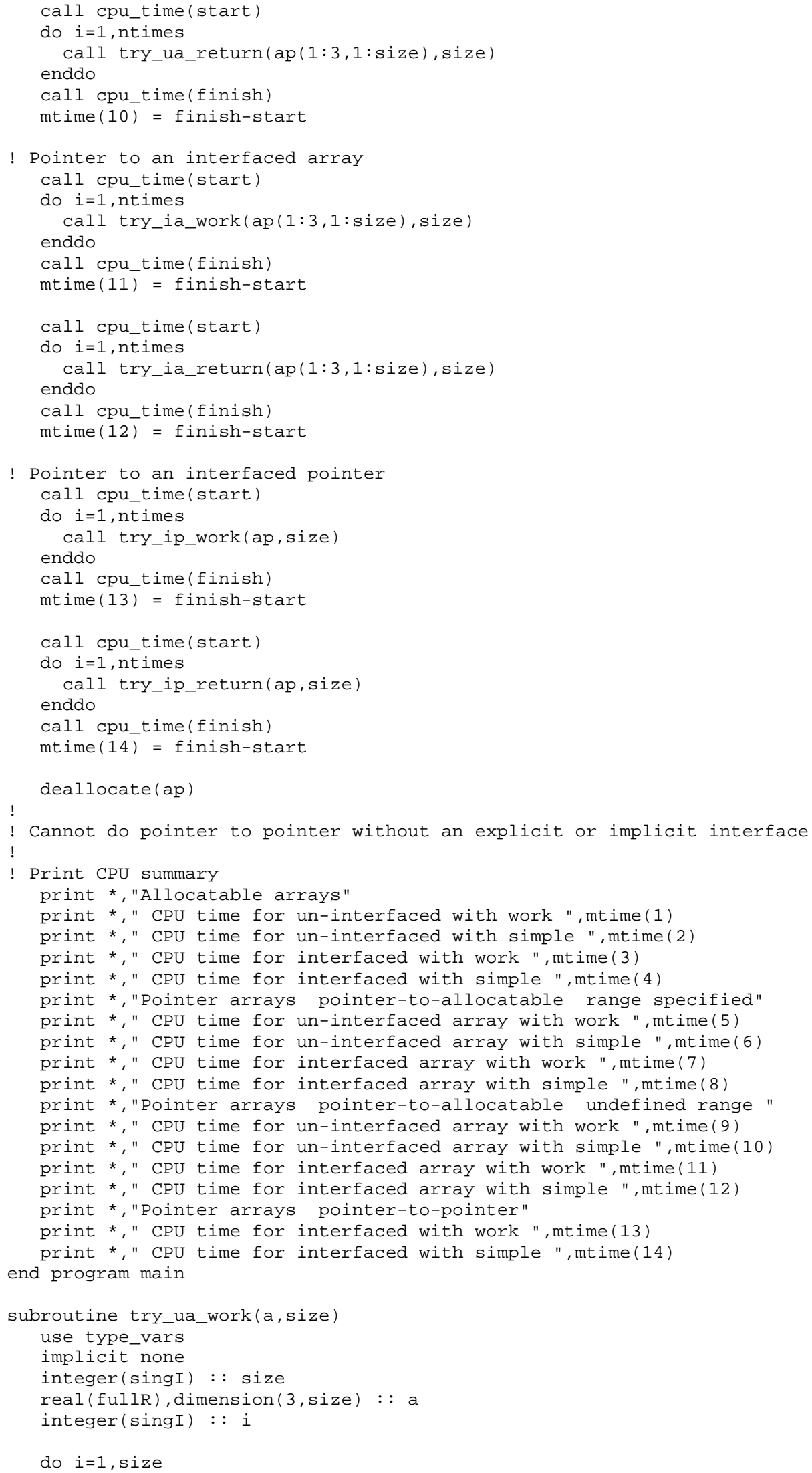




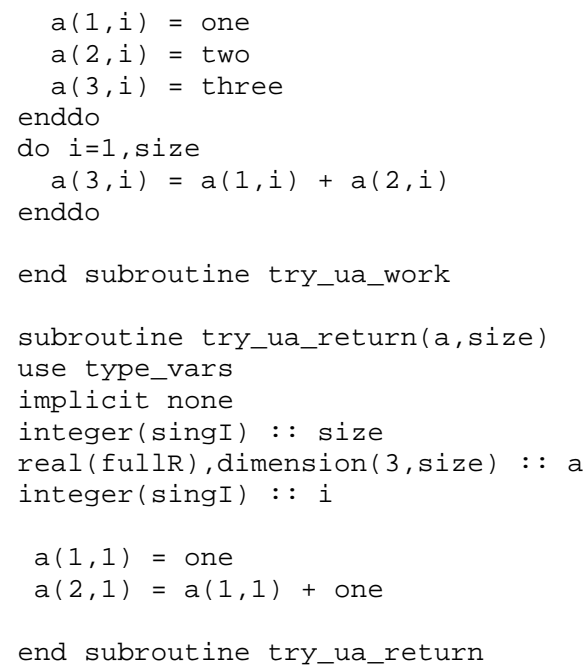

